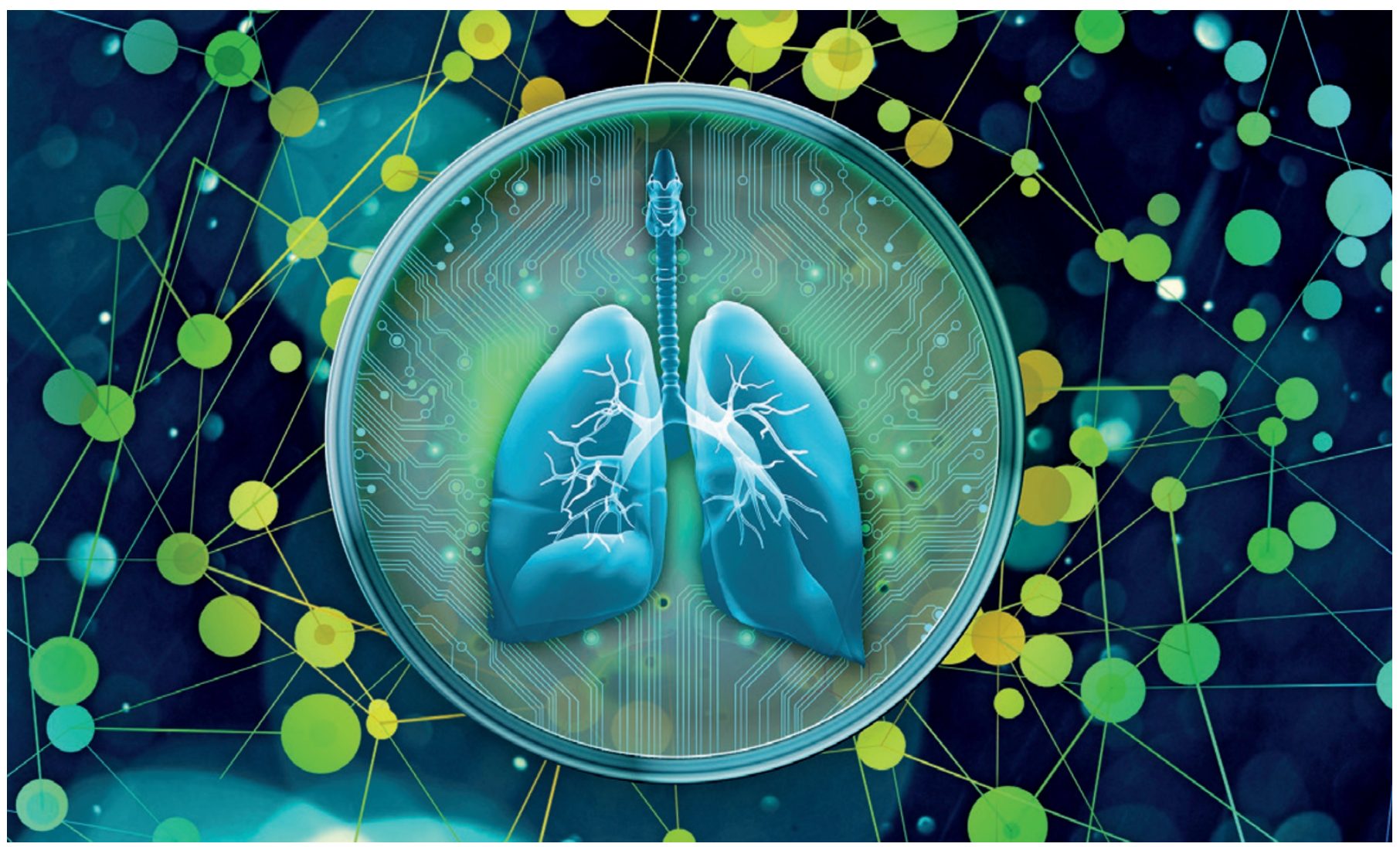

ARTIFICIAL ORGANS

\title{
Honey, I shrunk the lungs
}

\section{Miniature versions of hearts, lungs and other organs are heralding a bright future for drug research and discovery.}

\section{BY MICHAEL EISENSTEIN}

I n 1537, the Swiss German scientist Paracelsus conceived a method for creating a miniature person who "will look somewhat like a man, but transparent, without a body". However, his protocol for generating what he dubbed a homunculus was an unsavoury blend of alchemy and inter-species breeding.

Now scientists are having another attempt at the homunculus concept - but this time they are using advanced biomaterials, engineering techniques and physiological insights to coax cells to grow into simplified, but functional, miniature versions of hearts, lungs and other human organs. Set on microchip-like devices, several of these organs can be connected by artificial circulatory systems to yield 'humanon-a-chip' devices that usually measure no more than a few centimetres in length.

The goal is to 're-humanize' biomedical research. For generations, scientists have worked mainly with cells grown in single layers on flat, artificial surfaces - typically hard plastics such as polycarbonate that bear no resemblance to living material - in pools of synthetic cell-culture media comprising cocktails of only the most essential ions, nutrients and growth factors.

\section{A DIFFERENT APPROACH}

In contrast, the bioengineered homunculi that researchers such as Donald Ingber, founding director of Harvard University's Wyss Institute for Biologically Inspired Engineering in Boston, Massachusetts, and his colleagues are devising aim to recreate the physical structures, forces and interactions that affect cells within living organs and tissues. The resulting experimental models are more realistic than conventional cell culture, more relevant than laboratory animals, but still small enough to fit under a microscope. They promise to eliminate a lot of the false starts and dead ends in the challenging and expensive world of drug discovery and testing (see 'Conceptual view of a human-on-a-chip').
The current system of drug development, which requires multiple rounds of animal testing, can lead to problems because humans often metabolize chemical compounds very differently from rodents and other test animals. For example, troglitazone was initially approved in the mid-1990s for treating diabetes in the United States, Japan and the United Kingdom, but was withdrawn in 2000 after causing liver failure in hundreds of patients including 63 deaths. The toxicity did not show up in earlier rat experiments.

Actual human tissues can be used to complement animal testing, but tissue slices are shortlived and hard to come by. Researchers can also extract primary cells, such as liver hepatocytes, from the relevant human organs but these gradually lose their functional identity when cultured on flat plastic. Even more daunting is the fact that it is difficult to recreate in cultures the complex interplay between communities of multiple cell types that enable an organ to function, says John Wikswo, a physicist and biomedical engineer at Vanderbilt University 
in Nashville, Tennessee. "Heterogeneity is a big deal," he says.

Advances in biomaterials now enable scientists to begin mimicking this organization by taking advantage of how cells naturally arrange themselves within nests of protein fibres known as the extracellular matrix. These fibres not only provide a physical scaffold, but also interact with receptors on the surface of the cells to trigger essential signals that regulate cell growth and development. Although the natural structure of the extracellular matrix is difficult to replicate, simply coating plasticware with a carpet of extracellular matrix proteins can give cultured cells a comfortable surface to latch on to and help to restore some of their capacity for self-organization.

\section{FORCES OF NATURE}

Sangeeta Bhatia's biomedical engineering team at the Massachusetts Institute of Technology (MIT) in Cambridge developed a greatly improved liver culture model ${ }^{1}$ by depositing human hepatocytes onto islands of the extracellular matrix protein collagen. These were then surrounded by other cell types, such as skin fibroblasts, which interact with these hepatocyte islands to help maintain liver-specific metabolic function and gene-activity profiles that would otherwise be lost. "We can basically create a miniature human liver on a dish," says Bhatia. This has enabled her team to study liver diseases caused by human-specific pathogens that are extremely challenging to model in animals, such as hepatitis and malaria.

These culture systems still lack the multilayered three-dimensionality of real organs, a feature that can greatly affect the behaviour of cells within a tissue. Researchers are therefore attempting to make the systems more realistic by endowing them with more volume using specialized materials and even cell-printing processes to construct solid volumes of organized, cultured cells. Various water-based hydrogel polymers can be coaxed from a liquid

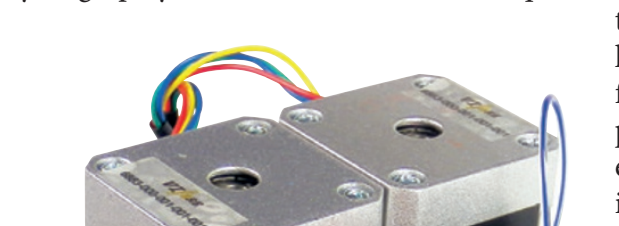

to a solid state by changing the $\mathrm{pH}$ or by using lasers, allowing scientists to entrap defined cell populations within solid matrices. Bhatia's inVERT technique ${ }^{2}$ uses moulds to generate three-dimensional (3D) patterns of hydrogelencased cells, which can then be overlaid with additional layers of cells - sequentially assembling different cell types into highly designed, customized tissue communities in a manner similar to the way in which a printmaker adds layer after layer of stencilled colours to produce a complete artwork.

These 3D systems may be more physiologically accurate than conventional Petri cultures, but they still fail to recapitulate many core organ functions because they are static. Within the dynamic environment of the body, physical forces create pres-

"We can basically create a miniature human liver on a dish." sure and tension on envi-

ronment-sensing cell-surface proteins. In a process known as mechanotransduction, those physical forces generate biochemical signals that can trigger dramatic changes in cellular organization and behaviour. The most universal of these is the shear force generated by blood flow; the endothelial cells that line blood vessels form a chaotic sprawl in static culture, but reorganize and align when subjected to directional fluid flow. The bioreactor systems that scientists have long used to replicate such flow are costly and bulky. As an alternative, techniques and tools developed for silicon-chip construction are being adapted to create scaled-down bioreactors based on microfluidics - glass-andpolymer-based devices in which tiny pumps, valves and channels direct liquids similarly to how a microchip moves electrons.

Organ-on-a-chip systems can also harness other biologically relevant forces, which is especially evident in the work of researchers such as Ingber. To mimic the conditions of the lung within a microfluidic device, Ingber's team cultivated epithelial cells derived from human alveoli and endothelial cells derived from blood vessels on either side of a porous polymer membrane. The cells on one side were exposed to air; those on the other side to flowing liquid. By periodically applying a vacuum

to adjacent cavities in the chip, the scientists could cause these membrane-bound cell sheets to stretch and relax, mirroring the rhythmic strain of breathing. This artificial lung reacted in a humanlike way when exposed to bacteria and to inflammation-causing nanoparticles, and allowed Ingber's

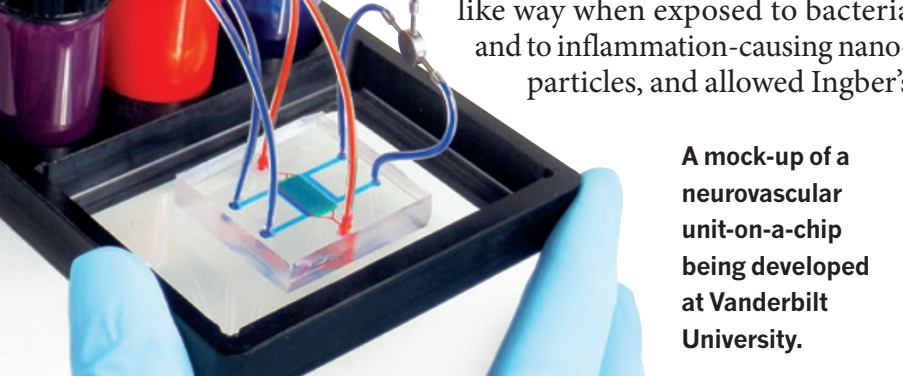

team to explore the mechanisms underlying fluid accumulation within the lungs of people with pulmonary oedema ${ }^{3}$. By selectively applying physical forces in culture models, his group has also been able to re-enact the realworld function of a host of other organ systems using just a handful of cell types. "All we do is change the physical microenvironment and we observe behaviours that nobody else sees," he says. "We saw the same thing in models of the gut with peristalsis and the kidney with liquid flow, and we were surprised by the level of fidelity and robustness."

\section{COMMUNITY ORGANIZERS}

Researchers say that the greatest promise for this kind of technology will come when organs-on-a-chip are integrated into simplified simulations of the body. This might yield significant benefits in drug testing, where the full effects of a compound are often not clear until processing in the liver or excretion from the kidney is accounted for.

Michael Shuler, a biomedical engineer at Cornell University in Ithaca, New York, was among the pioneers in this field. Shuler's team analyses the toxicity of various chemicals using what Shuler calls a microscale cell-culture analogue device, which steadily pumps cell-culture media between culture compartments representing different mammalian organ systems. As an initial test, the team used the household chemical naphthalene (the active ingredient in mothballs) in a multi-organ device containing lung, liver and fat tissue. The study revealed how naphthalene is converted by the liver into metabolites that cause cell damage and death in the lung - even though naphthalene itself is relatively harmless to this organ. "If we removed the liver component we no longer observed this cell death in the lung tissue," says Shuler. Other multi-organ systems followed, evolving from two-dimensional (2D) cultures on porous membranes to more complex aggregates within $3 \mathrm{D}$ hydrogels and other matrices.

At the Wyss, Ingber and his colleagues are in the process of linking up large numbers of their miniaturized organs; in February 2014, they reached a key milestone of building an automated system that could keep a ten-organ chip alive and functional for longer than a week. Ingber, Shuler and others have also shown that simpler multi- or single-organ constructs can be sustained for up to a month - sufficient for analysing toxicity caused by long-term exposure to a chemical or product.

But linking multiple organs is not as simple as connecting biological Lego. Some of the biggest challenges involve scaling in vitro systems to match human physiology ${ }^{4}$, such as maintaining proper fluid volume relative to tissue volume. Microfluidic systems that stream small quantities of culture media through narrow channels containing 3D cell structures can mirror real life better than large-volume culture systems, but these systems must still 


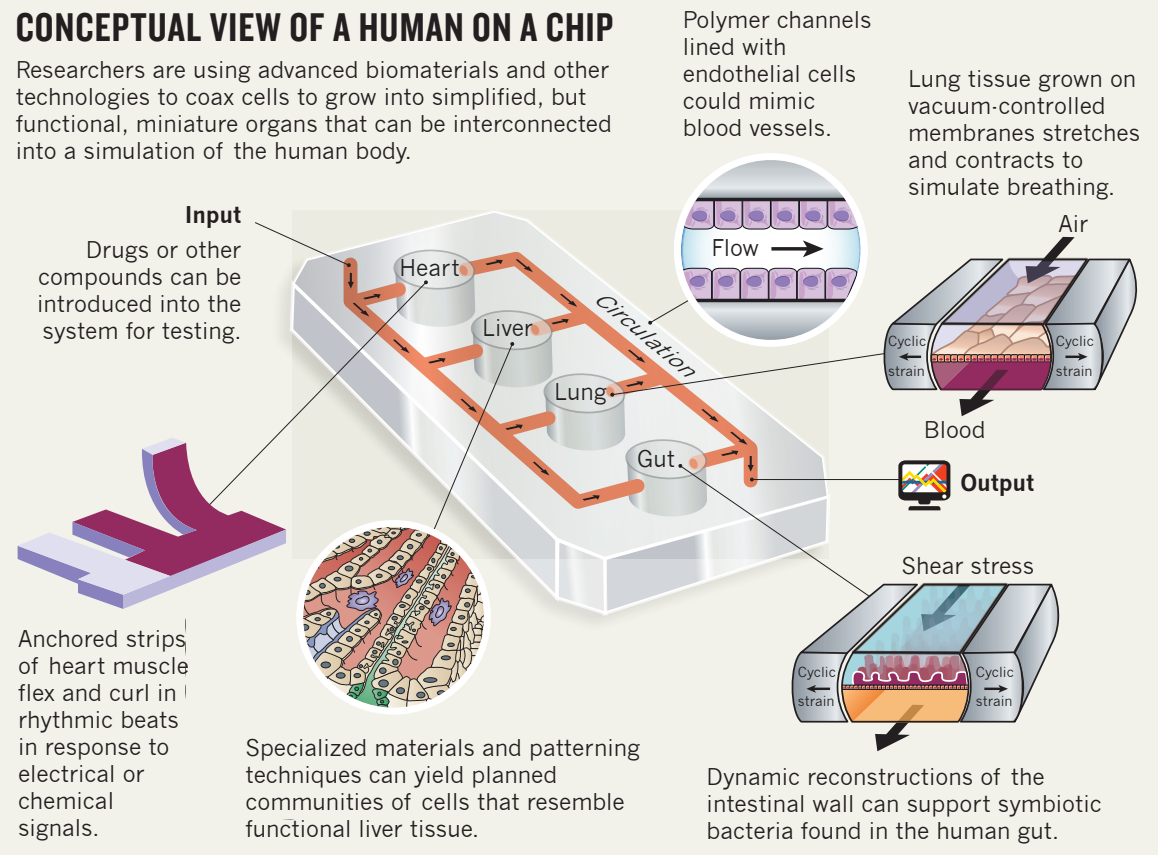

be carefully designed. "If you get the volumes wrong, suddenly the liver and the kidney are each swimming in oceans of media and they don't know that the other organ exists," says Wikswo. Relative size also matters: building a miniature human body-on-a-chip does not merely involve shrinking every organ 1,000 fold. Organ size is determined by diverse factors, including an organism's mass, surface area and fluid volume. For simplicity's sake, many researchers are looking to scale organs on a functional basis to preserve their relative physiological output, with tissue compartments sized such that the simulated lung draws in the appropriate amount of oxygen with each breath, and the liver metabolizes biomolecules at a proportional rate. "I essentially treat each organ as a chemical reactor," says Shuler.

Substrate materials also remain a challenge. Most organ- and body-on-a-chip devices are constructed from polydimethylsiloxane (PDMS), a polymer that is cheap, transparent, non-toxic and easily developed into prototypes. But PDMS also absorbs small molecules — including, unfortunately, many drugs — so the hunt is on for more suitable polymers. One alternative would be to connect various organ compartments using bioengineered blood vessels that line the chip's polymer channels. In autumn 2014, Bhatia collaborated with Christopher Chen of Boston University in Massachusetts, Gordana Vunjak-Novakovic of Columbia University in New York and Karen Hirschi of Yale School of Medicine in New Haven, Connecticut, to develop such a multi-organ chip, in which heart and liver compartments are joined by living capillaries. Uwe Marx of the Technical University of Berlin says that this will add a crucial dimension of realism. "The cells in these vessels are also transmitting information between the tissues," he says. "The blood-vessel system is about a lot more than just providing nourishment for organs."

It still remains unclear exactly what should be pumped through these vessels; although every tissue in our body is supported by a common fluid - blood - the search is still underway for a blood surrogate that many different cultured cell types find universally agreeable. "We may end up using the same fluid throughout the chip, but locally supplementing each compartment with distinct growth factors," says Bhatia. "That's kind of how the body operates." If lung tissue requires a biomolecule that adversely affects liver or other cell types, for example, it could be possible to limit its area of effect by storing it in a hydrogel repository that slowly releases the factor into media entering the lung compartment.

\section{SIMPLE, BUT NOT TOO SIMPLE}

Ingber emphasizes that his primary goal is to mirror function, not structure. "We start at the simplest embodiment and see what it can do," he says. "If it's missing something, we add complexity one step at a time, and if not we've just learned that we don't need that."

However, he also emphasizes that his group is pursuing the lofty goal of constructing a representation of the human body with the greatest possible functionality, in partnership with Wyss colleague Kevin Parker. It would have a beating heart, churning guts and breathing lungs, all supported by an automated system. This may be more sophisticated than many research groups require. Wikswo's group, in contrast, is looking into simpler arrangements. "The ultimate way to exploit these systems," he says, "will be to get small, cheap gadgets in the hands of any biologist who needs them."
Many labs in this space have created spin-off companies to develop their platforms; Marx co-founded the Berlin-based TissUse in 2010, which now offers two-tissue chips for drug and toxicology testing. Ingber and Shuler have also launched start-ups. In parallel, the US National Institutes of Health and the Defense Advanced Research Programs Agency have committed nearly US\$150 million to a cross-agency programme funding development of single-organ and body-on-a-chip devices. The US Food and Drug Administration has been actively involved as well, exploring how these systems might fit into their regulatory processes.

There is general agreement that by offering a true window into the body's inner workings, these compact, transparent organ-on-a-chip systems could transform drug testing. It is possible to incorporate
"I essentially treat each organ as a reactor." chemical sensors that track metabolism using glucose levels and $\mathrm{pH}$, or precisely measure the strain of contracting muscle or the flow of blood in real time. This could, for example, show whether a drug has the potential to alter a patient's heart rate or interfere with liver function. Wikswo is collaborating with Vanderbilt chemist John McLean to directly integrate molecular analysis using mass spectrometry, quantifying thousands of biomolecules within tiny volumes at ten-minute intervals. Simulated organs can even be removed for closer histological examination, and then replaced without disrupting experiments.

"The goal now is to learn and understand the questions that could not be solved with animals or with any other single organ or single tissue culture solution so far," says Marx. Beyond sparing myriad lab animals, these devices could also greatly streamline human trials for example, evaluating preclinical candidates so that only the best proceed to phase I. "You can test five to ten related drugs on microhuman chips and then send one of those forward," says Wikswo. "Then if something interesting — good or bad - happens during phase I, you can wheel these devices back out to figure out what's happening."

The broad hope, Wikswo says, is that biomedical research will benefit from a shift based on static cultures to dynamic platforms that generate steady streams of physiological data. "We want it reliable enough that it works for the experiment, small enough that you can fit 20 of them in an incubator and cheap enough that you can throw it out afterward."

Michael Eisenstein is a freelance science writer in Philadelphia, Pennsylvania.

1. Khetani, S. R. \& Bhatia, S. N. Nature Biotechnol. 26 120-126 (2008).

2. Stevens, K. R. et al. Nature Commun. 4, 1847 (2013).

3. Huh, D. et al. Science Transl. Med. 4, 159ra147 (2012)

4. Wikswo, J. P. et al. Lab Chip. 13, 3496-3511 (2013). 